\title{
Human-Centered Design for Advanced Technology
}

\author{
Satoshi Muraki \\ Department of Human Science, \\ Faculty of Design, Kyushu University,Japan \\ muraki@design.kyushu-u.ac.jp
}

\begin{abstract}
The development of technology has contributed to and changed almost all aspects in human life. However there are some inevitable negative consequences that come along with these massive changes. This paper discusses the aspects changed or influenced by the technological advances and how designers could contribute positively amids the rapidly growing phenomena. Designs that bridge the coexistence between technology and human values are needed to guide the advances into a direction that lead to better future.
\end{abstract}

Keywords: Advanced technology, human centered design, impacts of technology, coexistence

\section{INTRODUCTION}

Today's human society, culture, and civilization have developed alongside highly advanced technology. In the latter part of the 20th century, technology contributed to increased efficiency, saving of tasks, and environmental comfort in our daily lives and work. However, technology has also caused various harmful consequences in our society. This is particularly true of developed countries. Against this background, new concepts, including, "technostress" and "techno-adaptability" were presented by the academies to warn about the harmful effects of such advances [1].

In the 21st century, technological advancement has been rapidly accelerated, which has led to dramatic changes in human lifestyles and working styles. Today, people worldwide, irrespective of age, daily use the Internet and smartphones. These facilitate general people to easily undergo various new advanced technologies such as artificial intelligence (AI), internet of things (IoT), artificial

virtual spaces, etc. In addition, worldwide attention is attracted to novel and advanced technologies, including automatic drive vehicles, wearable devices, robots, humanoids, assistive/powered suits, and new style interfaces operated by gestures, voice commands, and even brain signals.

So far, technology has contributed to such various fields as health care, medicine, care, hygiene, labor, transportation, communication, education, art, amusement, security, and disaster prevention/recovery. Further successful advances are predicted to emerge quite soon. The development of technology must continue in the future, despite the fact that it has had harmful effects upon as well as provided boons to human beings as mentioned above. Here, what can designers and researchers of human science do to lead the technological advances to the right direction? In my keynote speech, I will review concerned disadvantages of the advanced technology, which relates directly and closely to human daily lives and working, and propose how to manage these issues occurring in the present and future from viewpoints of human-centered design.

\section{DEMERITS OF ADVANCED TECHNOLOGY}

\section{A. Health}

A smartphone is one of reprehensive advanced technology in the modern society. In 2017, the number of smartphones shipped throughout the world was over 1.5 billions [2]. This statistic indicates that the smartphone is an indispensable product for human daily life today all over the world. The spread of smartphones with the Internet has created various new styles of social network communication, which increase the number of heavy users.

Many studies have recently reported that prolonged smartphone exposure brings harmful mental and physical health problems such as straight neck, texting-related thumb injuries, vision loss, sleep disorders owing to the blue light of smartphone screens, and even depression [36]. Furthermore, such harmful effects spread to not only mental and physical health problems but also various aspects including weak human relationships, low recognition of difference between reality and virtual reality, low thinking skills, and low morality. Among high school students in Japan, for example, average daily smartphone use was 4.8 among males and 6.1 hours among females, respectively [7], which indicates a pervasive level of smartphone addiction that diverts them from important matters such as study, communication with family, and sleep.

The increase in the use of computers by individual at the workplace as well as at home has led to prolonged hours of sitting and looking at a monitor and awful posture of upper arms have resulted in various health problems such as back pain, stiff neck, carpal tunnel syndrome, and eye disorders. Furthermore, computers and other technologies have decreased opportunities for daily physical activity and a resultant increase in the prevalence of illness caused by insufficient exercise

\section{B. Humanity}

In 2014, an 89-year-old British woman committed suicide at Dignitas (a Swiss suicide clinic) for the sensational reason that she "cannot adapt to modern times" in which "People are becoming more and more remote," as if they were "becoming robots" with a "lack of humanity" [8]. Our technology has enabled us to communicate with anybody in the world through various 
communication styles including SNS, text chat mail, video calls, and more. For contemporary people, however, opportunities for commutation by face to face and mutual support in the community have been markedly reduced. Furthermore, robots and intelligent machines as well as Internet technologies provide convenient, comfortable services and otherwise inaccessible information to human beings. However, like the unfortunate British woman mentioned above, we sometimes feel a lack of humanity in our lives, inundated as they are by technologies, because we cannot directly touch human kindness, warmth, love, etc. through technology.

\section{Employment}

In societies that experience labor shortage, advanced technology is expected to contribute to fill the gap. For instance, in countries with high and increasing aged populations, particularly the elderly in need of care, care facilities and nursing homes need an increasingly greater number of staff members to care for them. In Japan, because the productive age population (15-65) is rapidly decreasing, most facilities for the aged face human resource shortages. Accordingly, advanced technologies such as nursing care robots are greatly expected to make up for such shortages.

In near future, it is said that various jobs will be superseded by AI and robots [9-10]. This means that some workers would lose or be forced to change jobs. As technology advances, workers are required to have an increasingly minimal amount of knowledge and technical skills. This requirement would place the middle aged and elderly in an unfavorable position as it would be difficult for them to be trained to perform. It is necessary for our society to create new jobs related to advanced technology that do not require advanced knowledge and technique.

\section{Cultivated skills and know-how}

Human society has handed down various forms of knowledge, experience, wisdom, know-how, and skill from generation to generation, further developed them, and consequently developed their culture and civilization throughout history. However, the advent of new technology sometimes reduces their usage and is replaced by new know-how and skills. For example, the motor-car was born in the 18th century and human society has continued to develop the motor-car self as well as traffic environment, rules/laws, education for driving skill and traffic safety, and so forth, since then. Throughout, people had to learn necessary driving skills and acquire driving licenses to drive a motor car on public roads. However, if automatic driving technology is completely achieved, we will not need driving skills, let alone a license to drive a motor-car, and their cultivated skill and know-how will no longer be useful and the society must establish new social system/environment and rules/laws for it.

In the future, these kinds of social change might occur through various technological advances such as automatic translation, nursing care robots, and so forth. As technology enacts various activities traditionally performed by human beings, knowledge and skills will decrease or shift. This involves extreme risks for society. If the spread and acceptance of technologies in society were suddenly paralyzed due to some reasons like a cataclysmic disaster or mass social unrest, with the result that jobs that people rely upon but can no longer perform capably owing to technological dependence, society and our lives will be thrown into chaos. We are in a period in which we have to decide from what until what we wish to rely on advanced technology in our lives, work, and society.

\section{E. Adaptability}

Recent technological advances can control various environmental factors such as temperature, humidity, airflow, air-component, air-pressure, sound, smell, and light with high precision, giving a comfortable environment to modern human beings. However, they have various potential adaptability to maintain their homeostasis of a living body against physical, chemical, and biological stressors within a natural environment such as cold, hot, wet, dry, high/low pressure, night and day cycle, and so forth. Habitual life at a comfortable environment decreases opportunity for activation of those homeostasis functions, which deteriorates them according to the biological principle.

Lately, abnormal whether events have more frequently occurred, such as intense heat waves and extreme cold. Artificially controlled environments through technology are useful for preventing health problems caused by severe climatic environment. However, it is important for us to maintain this basic potential adaptability against environmental changes without relying on artificial environment.

\section{F. Role of elderly people}

At the Second World Assembly on Aging in 2002, Kofi Annan, then-Secretary-General of the United Nations, stated in his address that "In Africa, it is said that when an old man dies, a library vanishes. The proverb may vary among continents, but its meaning is equally true in any culture. Older persons are intermediaries between the past, the present and the future. Their wisdom and experience form a veritable lifeline in society" [11]. As Annan mentioned here, the elderly have acquired precious experiences, knowledge, know-how, and wisdom throughout their long lives, which had contributed to the development of society in each age and had been their main roles throughout human history. Yet in the digital age, we often search for necessary information easily through the Internet without relying on the elderly's acquired wisdom. The development of technology forces both present and future seniors to find new social roles.

\section{G. Culture and tradition}

Each community (country, local region) has a unique culture and a unique tradition that have been developed through history. They are points of pride and spiritual support for the people in the communities. Development of the Internet has dramatically changed the shape of communication and information all over the world. This has enabled people to get a variety of information about other communities easily and quickly. In other words, people from one community can easily touch new and/or different ways thinking and values from another. Yet despite the social and cultural changes 
wrought by a global information society, we must think of ways to preserve our cultures and traditions and/or combine the existing culture and tradition with advanced technology.

\section{H. Child development}

The period from infancy to early childhood is of paramount important for the healthy growth and development of a human being's mind and body. The stimulus received during this period strongly influences later health and also personality traits such as character, thinking, and values. However, in contemporary society, even babies and other very young children often touch modern devices such as smartphones and tablet devices [12]. In fact, many parents in most countries have already accepted smartphone use by their babies and children in order to reduce their burdens in raising them. We therefore have to think about ways of promoting preferable patterns of growth and child development from infancy in a technologically advanced society.

\section{Distinguishing between real and virtual reality}

Recently, various $\mathrm{X}$ reality technologies including virtual reality (VR), augmented reality (AR), mixed reality (MR), substitutional reality (SR) have been developed. Some realities have been already in practical use, especially in the entertainment and game industries. Whereas these technologies have been expected to contribute to business (e.g., virtual shopping), education, medicine, training in special skills, they also cause various troubles. For example, it would appear that people sincerely and seriously love the virtual humans that they encounter in virtual reality and cannot love real humans. In addition, some people have become so immersed in virtual reality that they cannot distinguish between real and virtual life. This could lead to crimes and violence in real society. In the near future, it is predicted that we may not distinguish between real and virtual reality due to the extremely high quality of the latter.

\section{J. Etc.}

Here, various expected demerits of the advanced technology are summarized. Those should be just little parts of them. We must always keep in mind that technology has merits and demerits.

\section{USER-ORIENTED DESIGN AND TECHNOLOGY- ORIENTED DESIGN}

In Japan, advanced technology has been strongly expected to solve serious social problems mainly due to the declining birthrate and the aging population, both of which are decreasing the working population. For example, most nursing home staff members have lower back pain due to heavy manual work [13], which is one of the main reasons they resign. Accordingly, the development of new technology has been expected to assist human careworkers in, for example, lifting a person in need of care. In addition, in order to decrease the number of people in need of care and enhance the selfsupporting (independence) ability, new technology has been also expected to assist the aged and infirm with daily physical activities like walking, standing, sitting, and eating [14].
However, advanced technologies have been not always accepted in human lives and their work. For example, the development of assistive devices enabling human motion sometimes originates through technical seeds; in other words, an engineer's motivation. This starting process is called "technology oriented." It has been pointed out that the products developed by this process sometimes do not meet the user's needs. For example, if the performance of enabling motion is excellent but are accompanied by some problems (e.g., duration and difficulties putting on and taking off a device, disadvantages for other motions, etc.) users do not accept the products. When new assistive products are developed for care workers and elderly people, we need to consider whether targeted users are receptive to including them in their lives and whether the assistive products work from a user's perspective.

\section{HUMAN-CENTERED DESIGN FOR ADVANCED TECHNOLOGY}

A. Co-existence with technology

Although the technology has brought various harmful effects on human beings as mentioned above, its growth cannot be stopped because human desires are limitless as the human history proves, and technological advancement attempts to meet these desires. Historically, when new technologies were introduced into a society, their merits were mainly emphasized by developers/engineers and their related organizations. Thereafter, their demerits were recognized and pointed out by users. Then, related academic societies start to deal with their problems and propose their countermeasures to prevent their demerits. From now on, before we introduce a new technology into the society, we have to estimate its possible harmful effects, propose effective measures to the concerned people and organization, and raise the alarm to the society.

Furthermore, artificial robots, AI-related products, wearable devices, and intangible interfaces will be widely used in not only the workplace but also in common households. This entails that everybody will daily use them, irrespective of age, sex, or occupation. However, the acceptance of these technologies must be different from individual to individual. Recently, commutation robots giving opportunities of daily verbal conversation have been spread especially for elderly living alone. Although these are useful for some of elderly to find fulfillment in their daily lives, some of them lose interest. This phenomenon is related to users-individual characteristics including their own values and past experiences with technology. Based on differences among individuals' respective levels of acceptance of advanced technology, it is important to think about how the technology should be developed and spread throughout human daily life.

\section{B. Understanding human adaptability to new advanced technology.}

So far, technology has created artificial environments to control various environmental factors such as temperature, humidity, and air quality. They have expanded human activities of life and work both quantitatively and qualitatively. For example, air conditioners can provide comfortable thermal 
environments for living and working as well as compensate weakened thermoregulatory functions in the elderly and people with disabilities. Thus, human beings have created an artificially controlled environment around them, which in turn has expanded human activities.

On the other hand, new advanced technologies have recently been becoming direct extensions of or substitutes for human functions such as sensory, information processing, and motor functions. This is so-called "superhuman." For example, assistive/powered suits recently developed try directly to act human joint movements by advanced sensing and motor controlling technology. These technologies are expected to contribute to decrease human levels of hard physical/manual labor in factories, construction sites, agriculture, forestry, and fishing, as well as in nursing homes to increase motion ability in elderly people with weak muscles or who suffer imbalances or mobility impairment. In addition, these technologies are expected to maintain and improve the ability of those people. In the near future, these kind of technologies are projected to become familiar with human living and working.

So far, in the field of care and rehabilitation, there are many products (ordinary care devices) that compensate for the weakened motion ability of elderly and infirm users, including manual/electric wheelchair, nursing bed, electric standing devices, machines to transfer individuals into automobiles, and more. These devices act almost completely as weakened motion function, and their function does not need to work. In contrast, those some kinds of new assistive technology such as assistive/powered suits require users to engage in unique cooperation movement with the machine [15]. In order to improve effective output, namely, better performance of the movement, users have to perceive assistive force through sensory functions and to release or adjust their muscle tension skillfully to use the mechanical force from the machine. In other words, these new technologies require new original and unique skills to human beings. Therefore, it is crucial to investigate humanity's potential ability and adaptability to new advances in assistive technology and to apply their findings to the development of human-centered assistive technology.

\section{Co-existence between nature and technology.}

In the entire sweep of human history, the period of the advanced technology and civilized society has existed for only an extremely brief moment compared with the long period of hunter-gathering societies in the more than two million years of homo species. Throughout evolutionary history, human beings have adapted to severe nature environments through biological and cultural strategies.

However, the contemporary living environment is markedly different from that in of our ancestors lived. Although various artificial living environments provided through the advanced technology bring many merits such as a comfortable life, they also bring various new mental and physical stress--in other words, modern diseases. The more civilization and technology advances, the more people might long for nature. It is important to think about ways of creating a society that co-exists with nature and technology in a harmonious balance.

\section{RELATED ACADEMIC FIELDS}

In order to promote human-centered design for further advanced technology, two academic societies must play central roles, that is, "Ergonomics" and "Physiological Anthropology."

The International Association of Ergonomics, which is the premium authority on ergonomics in the world, defines it as "the scientific discipline concerned with the understanding of interactions among humans and other elements of a system, and the profession that applies theory, principles, data and methods to design in order to optimize human well-being and overall system performance." [16]. So far, ergonomics has been driving "human-centered design" mainly for products, jobs, environments, organizations, and tasks from viewpoints of human characteristics.

The International Association of Physiological Anthropology defines physiological anthropology as "the scientific discipline which focused on homo sapiens as the subjects existing in the modern technological society of today and aspired to the creation of living environments and lifestyle systems based on research which attaches importance to human characteristics evaluated from a viewpoint encompassing the past, present and future." [17]. This society has been challenged to study truly desirable human living and working conditions from the viewpoint of biological characteristics.

These societies have played various roles in solving the problems caused by modern technologies so far. Unfortunately, most of their strategies have been reactive actions, not proactive actions. These societies should be strongly required to predict problems facing humanity and the society occurring in the future, and then to steer advanced technology in the right direction for human beings [18].

\section{ACKNOWLEDGMENT}

This work was supported by JSPS KAKENHI (Grant No. JP 15K14619 and 17H01454).

\section{REFERENCES}

[1] K. Iwanaga, X. Liu, Y. Shimomura, and T. Katsuura, "Approach to human adaptability to stresses of city life," J. Physiol. Anthropol. Appl. Human. Sci., vol. 24, pp. 357-361, 2005.

[2] (2018 Aug.) IDC website [Online]. Available: https://www.idc.com/promo/smartphone-market-

share/vendor

[3] D. Sharan, M. Mohandoss, R. Ranganathan, and Jose $\mathrm{J}$, "Musculoskeletal disorders of the upper extremities due to extensive usage of hand held devices." Ann. Occup. Environ. Med., Vol26, 22. doi: 10.1186/s40557-0140022-3. eCollection, Aug. 2014.

[4] S. Lee, Y. Choi, and J. Kim. "Effects of the cervical flexion angle during smartphone use on muscle fatigue and pain in the cervical erector spinae and upper trapezius in normal adults in their 20s," J. Phys. Ther. Sci., vol.29, pp.921-923, May 2017.

[5] J.T. Dennerlein, "The state of ergonomics for mobile computing technology," Work., vol.52, pp.269-277, 2015. 
[6] J. Matar Boumosleh, and D. Jaalouk, "Depression, anxiety, and smartphone addiction in university studentsA cross sectional study," PLoS One, vol. 12(8): e0182239, doi: 10.1371/journal.pone.0182239, Aug 2017.

[7] (2018 Aug.) DigitalArts website [Online]. Available: https://www.daj.jp/company/release/2017/0301_01/

[8] (2018 Aug.) https://www.mirror.co.uk/news/uknews/teacher-committed-suicide-dignitas-after-3382668

[9] D.M. West, 'What happens if robots take the jobs? The impact of emerging technologies on employment and Assembly on Ageing," 2012, [Online]. Available: http://repository.forcedmigration.org/pdf/?pid=fmo:3660 [12] S. Yadav, P. Chakraborty, P. Mittal, and U. Arora, "Children aged 6-24 months like to watch YouTube videos but could not learn anything from them," Acta. Paediatr., vol.107, pp.1461-1466, Aug, 2018.

[13] K. Fusano, C. Kubo, K. Ozaki, K. Goto, and N. Tajima, "Risk factor on low back pain in the nurses and care workers". J. Lumbar. Spine. Disord., vol.13, pp.113120, 2017.

[14] J. Araullo, and L.E. Potter, "Promoting physical activity in seniors: Future opportunities with emerging technologies." Proceedings of the 2016 ACM SIGMIS Conference on Computers and People Research, pp. 5764, 2016.

[15] N. Nasir, K. Hayashi, P.Y. Loh, and S. Muraki, "The effect of assistive force on the agonist and antagonist muscles in elbow flexion" Malaysian Journal of Movement, Health \& Exercise, vol. 6, pp. 35-52, 2017.

[16] (2018, Aug.) IEA (International Ergonomics Association) website [Online]. Available: https://www.iea.cc/whats/index.html

[17] (2018, Aug.) IAPA (International Association of Physiological Anthropology) website [Online]. Available: http://www.intlphysiolanth.org/index.html

[18] S. Muraki, K. Iwakiri, "The current state and future of ergonomics in each research field," Jap. J. Ergon., vol.12, pp.79-85, 2014. public policy', Brookings Centre for Technology Innovation, Oct. 2015, [Online]. Available: https://www.brookings.edu/wpcontent/uploads/2016/06/robotwork.pdf [10] A. Smith, and J. Anderson, "AI, robotics, and the future of jobs," Pew Research Center 6, 2014. [Online]. Available: https://www.brookings.edu/wpcontent/uploads/2016/06/robotwork.pdf [11] United Nation, "Report of the Second World 